\title{
Misaponin B Induces G2/M Arrest, Cytokinesis Failure and Impairs Autophagy
}

\author{
Gunho Won, Ji Hoon Jung, Eun Jung Sohn (D), Ji Eon Park, Hyungin Kim, Hyo-Jung Lee, \\ Bum Sang Shim, and Sung-Hoon Kim
}

College of Korean Medicine, Kyung Hee University, Seoul 02447, Republic of Korea

Correspondence should be addressed to Sung-Hoon Kim; sungkim7@khu.ac.kr

Received 9 January 2019; Accepted 4 January 2020; Published 7 February 2020

Academic Editor: Nathalie M. Mazure

Copyright ( $) 2020$ Gunho Won et al. This is an open access article distributed under the Creative Commons Attribution License, which permits unrestricted use, distribution, and reproduction in any medium, provided the original work is properly cited.

\begin{abstract}
Saponins are a group of naturally occurring plant glycosides with the features of their strong foam-forming properties and multibiological effects such as antitumor activity. Though Misaponin B, one of the triterpenoid saponins from Madhuca longifolia, is known to have spermicidal and antioxidant activity, the other biological activities have been never reported so far. Thus, in the present study, the antitumor mechanism of Misaponin B was investigated in A549 and AsPC-1 cancer cells. Misaponin B exerted significant cytotoxicity in A549, H460, SKOV3, and AsPC-1 cancer cells. Among them, A549 and AsPC-1 cells were more susceptible to Misaponin B. Misaponin B induced G2/M arrest and cytokinesis failure and increased the expression of LC3B and p62 with autophagic vacuoles and GFP-LC3 punctae in A549 and AsPC-1 cells. Furthermore, Misaponin B suppressed autophagy flux in A549 cells transfected by GFP-mRFP-LC3 constructs by showing merged yellow color by autophagy flux assay. Overall, our findings provide evidences that Misaponin B induces G2M arrest and impairs autophagy in A549 and AsPC-1 cells.
\end{abstract}

\section{Introduction}

Though the war on cancer actively began with the National Cancer Act of 1971, cancer death rate still remains high so far [1]. Among many cancer therapies, the efficacy of chemotherapy in neoplastic patients is extremely limited, due to drug resistance and toxic side effects of drugs [2-4]. Thus, natural antitumor agents such as curcumin [5], decursin [6,7], and gallotannin are on the spotlight because of their weak toxicity and synergistic efficacy with classical anticancer drugs.

Autophagy is known as a self-degradative process in removing misfolded or aggregated proteins, clearing damaged organelles, such as mitochondria, endoplasmic reticulum and peroxisomes, and intracellular pathogens $[8,9]$. When autophagy, an intracellular degradation system, is activated in the cells, double-membrane autophagic vesicles (autophagosomes) formed to surround cytoplasm and/or cytoplasmic constituents and proteins are then fused with lysosomal vesicles to degrade the contents of the autophagic vesicle for complete autophagy [10-13]. In contrast, impaired autophagy, not leading to autophagolysosome, usually contributes the type 2 cell death known as autophagic cell death in all eukaryotic cells [14-17]. It is well documented that autophagic cell death plays an important role in the suppression of malignant tumors [17-19].

Emerging evidences reveal that some saponins including dioscins, saikosaponins, julibrosides, soy saponins, ginseng saponins, and avicins $[20,21]$ have antitumor effects in several cancers via regulation of apoptosis, autophagy, tumor microenvironment, and cell cycle arrest [22].

Misaponin B (MSB) is a novel triterpenoid saponin isolated from Bassia longifolia L. or Madhuca longifolia L. [23]. However, its biological effects were never reported except its spermicidal and antioxidant activity and our poster presentation [24]. Thus, in the present study, we report the underlying antitumor mechanism of Misaponin in A549 and ASPC1 cells in association with autophagic cell death.

\section{Materials and Methods}

2.1. Isolation of Misaponin B from Madhuca longifolia Seeds. The dried seed kernels $(100 \mathrm{~g})$ of $M$. longifolia were extracted with $80 \%$ aqueous methanol $(\mathrm{MeOH}, 500 \mathrm{~mL} \times 3)$, and the extracted solution was filtered and evaporated at $40^{\circ} \mathrm{C}$. The 
concentrate was transferred in water $(200 \mathrm{~mL})$ and consecutively extracted with ethyl acetate $(\mathrm{EtOAc}, 200 \mathrm{~mL} \times 3)$ and $n$ butanol $(n-\mathrm{BuOH}, 170 \mathrm{~mL} \times 3)$. The $n-\mathrm{BuOH}$ layer was concentrated to acquire residues of the $n-\mathrm{BuOH}$ fraction (MLB, $11.4 \mathrm{~g})$. The MLB fraction was poured to a Diaion HP column $(5 \mathrm{~cm} \times 20 \mathrm{~cm})$, eluting water $(500 \mathrm{~mL})$ and $\mathrm{MeOH}(800 \mathrm{~mL})$. The $\mathrm{MeOH}$ solution was concentrated at $40^{\circ} \mathrm{C}$ to give a crude saponin fraction (MLCS, $8.2 \mathrm{~g}$ ). A part of MLCS fraction ( $\mathrm{g}$ ) was subjected to silica gel column chromatography (c.c., $5 \mathrm{~cm} \times 10 \mathrm{~cm})$ and eluted with $\mathrm{CHCl}_{3}-\mathrm{MeOH}-\mathrm{H}_{2} \mathrm{O}(6: 4: 1$, $1250 \mathrm{~mL}$ ) to provide seven fractions (MLCS-1 to MLCS-7). Fraction MLCS-4 (375 mg) was applied to the silica gel c.c. $(3 \mathrm{~cm} \times 10 \mathrm{~cm})$ and eluted with $\mathrm{CHCl}_{3}-\mathrm{MeOH}-\mathrm{H}_{2} \mathrm{O}(8: 5: 1$, $450 \mathrm{~mL}$ ) to provide five fractions (MLCS-4-1 to MLCS-4-5) along with Misaponin B (MLCS-4-3, 187 mg). Misaponin B was identified on the basis of spectroscopic analyses such as NMR, $\mathrm{IR}$, and MS and stored at $-20^{\circ} \mathrm{C}$. The purity was $>90 \%$, as assessed by HPLC.

2.2. Cell Culture. Human A549, H460, SKOV3, and AsPC-1 cancer cells were purchased from American Type Culture Collection (ATCC, USA). The cells were cultured in RPMI1620 (WELGENE, Cat. LM 011-01, Korea) medium supplemented with $10 \%$ FBS and $1 \%$ antibiotic-antimytotic solution (WELGENE. Cat. LS203-01, Korea) at $37^{\circ} \mathrm{C}$ and $\mathrm{CO}_{2}$.

2.3. Cell Viability Assay. The cytotoxic effects of Misaponin $\mathrm{B}$ in A549, H460, SKOV3, and AsPC-1 cancer cells were evaluated by 3-(4,5-dimethylthiazol-2-yl)-2,5-diphenyltetrazolium bromide (MTT) assay. Cells were seeded onto 96well microplates at a density of $1 \times 10^{4}$ cell/well and treated with various concentrations of Misaponin B $(0,7.5,15,30$, 45 , or $60 \mu \mathrm{M})$ for $24 \mathrm{~h}$. The cells were incubated with 3-(4,5dimethylthiazol-2-yl)-2,5-diphenyl tetrazoliumbromide $(1 \mathrm{mg} / \mathrm{mL})$ (Sigma Chemical Co., USA) for $1 \mathrm{~h}$, and then optical density (OD) was measured using a microplate reader (Molecular Devices Co., USA) at $570 \mathrm{~nm}$. Cell viability was calculated as a percentage of viable cells in Misaponin B-treated group versus untreated control.

2.4. Cell Cycle Analysis. To perform cell cycle analysis, A549 and ASPC1 cells were treated with Misaponin B for $24 \mathrm{~h}$, collected, and fixed in 75\% ethanol. The fixed cells were then incubated at $37^{\circ} \mathrm{C}$ with $0.1 \%$ RNase $\mathrm{A}$ in PBS for $2 \mathrm{~h}$ and suspended in PBS containing $50 \mu \mathrm{g} / \mathrm{mL}$ PI for $30 \mathrm{~min}$ at room temperature. The stained cells were analyzed for DNA content in the FACSCalibur (Becton Dickinson, Franklin Lakes, NJ, USA) using the Cell Quest program (Becton Dickinson, USA).

2.5. Quantitative Real-Time PCR ( $q R T-P C R$ ) and Reverse Transcription PCR (RT-PCR). Total RNA was isolated from A549 and AsPC-1 cells with QIAzol (Invitrogen, USA). A reverse transcription kit (Promega, USA) was used to construct the template cDNA. qRT-PCR was performed with the LightCycler480 instrument (Roche Applied sciences, USA). GAPDH served as normalization control. Primer sequences used for PCR are as follows: LC3B forward 5'-AGACCTTCAAGCAGCGCCG-3', LC3B reverse 5'-ACACTGACAATTTCATCCCG-3'; miR1290 forward 5'-GAGCGTCACGTTGATCACGTTGACACT-3', reverse 5'-TTGAGCATCCCTGATCCA-3'; glyceraldehyde-3phosphate dehydrogenase $(\mathrm{GAPDH})$ forward $5^{\prime}$-TATA AATTGAGCCCGCAGCC- $3^{\prime}$, GAPDH reverse $5^{\prime}$-TTC CCGTTCTCAGCCTTGAC- $3^{\prime}$. The PCR was performed as follows: $5 \mathrm{~min}$ at $95^{\circ} \mathrm{C}, 30$ cycles of $95^{\circ} \mathrm{C}$ for $20 \mathrm{sec}, 55^{\circ} \mathrm{C}$ for $20 \mathrm{sec}, 72^{\circ} \mathrm{C}$ for $40 \mathrm{sec}$, and $5 \mathrm{~min}$ incubation at $72^{\circ} \mathrm{C}$. The PCR products were loaded onto $1 \%$ agarose gel electrophoresis and visualized by loading STAR solution (Dynebio, Korea) and UV illumination.

2.6. Western Blot Analysis. A549 and AsPC-1 cells were lysed in RIPA buffer (3 M, Lot\#R1140305) and were incubated on ice for $1 \mathrm{~h}$. After centrifugation, the protein contents were measured by using a Bio-Rad DC protein assay kit II. Proteins were separated by electrophoresis on $8 \%$ or $12 \%$ SDS-PAGE and electrotransferred onto a Hybond ECL transfer membrane. The membrane was blocked with $5 \%$ nonfat skim milk and probed with primary antibodies for LC3B, p62 (Cell Signaling Tech., USA), and $\beta$-actin (Santa Cruz Biotechnologies, USA), followed by incubating with horseradish peroxidase- (HRP-) conjugated secondary antibodies. Protein expression was detected by using an enhanced chemiluminescence (ECL) system (Amersham Pharmacia, USA).

2.7. Immunofluorescence. A549 and AsPC-1 cells were seeded on a chamber slide (Nalge Nunc. LAB-TEK. 177399, USA) and were then treated with $15 \mu \mathrm{M}$ or $30 \mu \mathrm{M}$ of Misaponin B for $24 \mathrm{~h}$. After washing, the cells were fixed with $4 \%$ paraformaldehyde in PBS for $20 \mathrm{~min}$, washed twice with PBS for $10 \mathrm{~min}$, and permeabilized by using $0.5 \%$ of Triton X-100 for $5 \mathrm{~min}$ at room temperature, followed by PBS with $2 \%$ bovine serum albumin (BSA) (AMRESCO. Albumin, Bovine. CAS\# 9048-46-8, USA) for $30 \mathrm{~min}$. The anti- $\alpha$-tubulin antibody was incubated. After washing twice with DPBS, A549 and AsPC-1 cells were incubated with Alexa 546 conjugated goat anti-rabbit IgG antibody for $1 \mathrm{~h}$. The chamber slide was mounted with a VECTASHIELD mounting medium with DAPI. Samples were imaged under a confocal fluorescence microscope (FV1000, Olympus, Japan).

2.8. Autophagy Flux Assay Using RFP-GFP-LC3 Construct. A549 cells incubated on a cell culture slide (SPL. Lot No. BA5A09A, Korea) were transfected with green fluorescent protein-labeled LC3 (GFP-LC3) or red-green fluorescent protein-labeled LC3 (RFP-GFP LC3) using X-treme GENE HP DNA Transfection Reagent (Roche, Lot. 11062400, Switzerland). The fluorescent color was analyzed in Misaponin B-treated A549 cells for $24 \mathrm{~h}$ by fluorescence microscopy. 


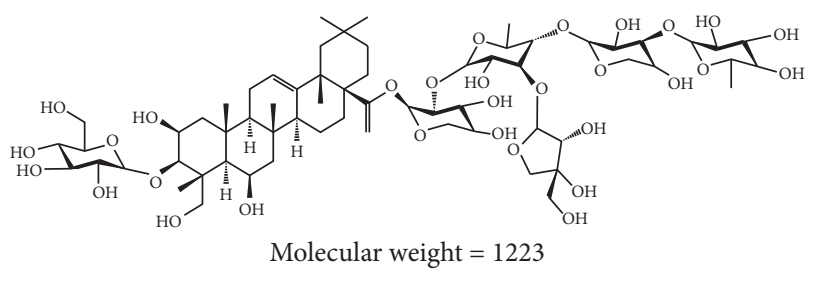

(a)
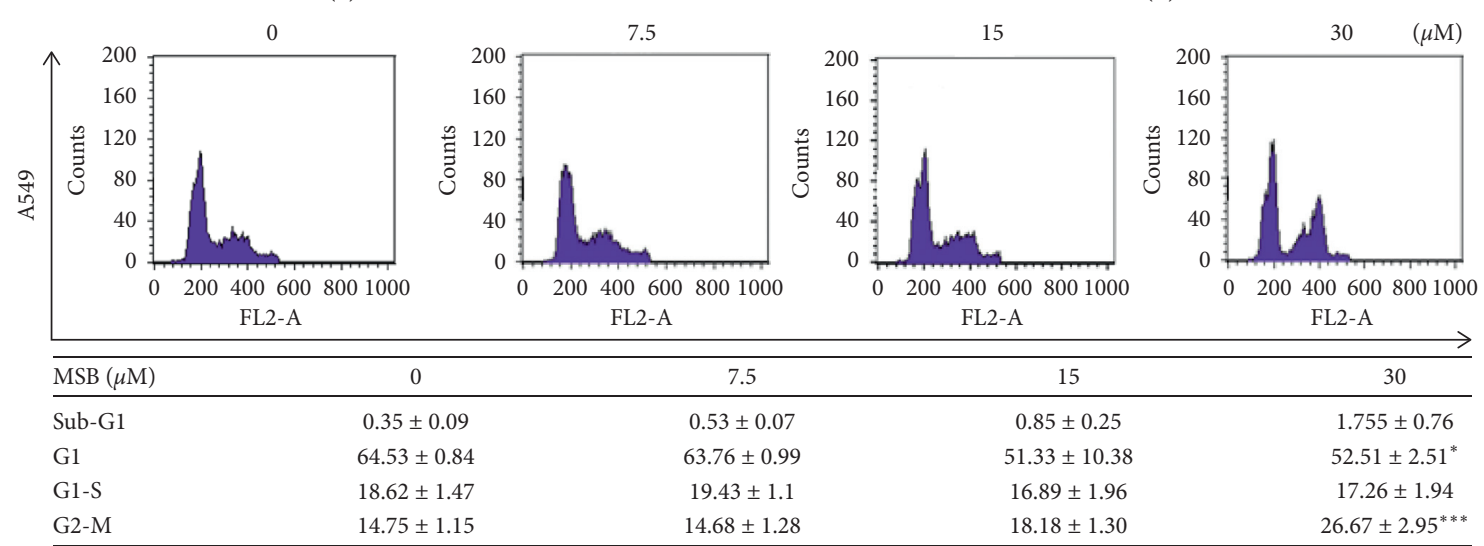

(c)
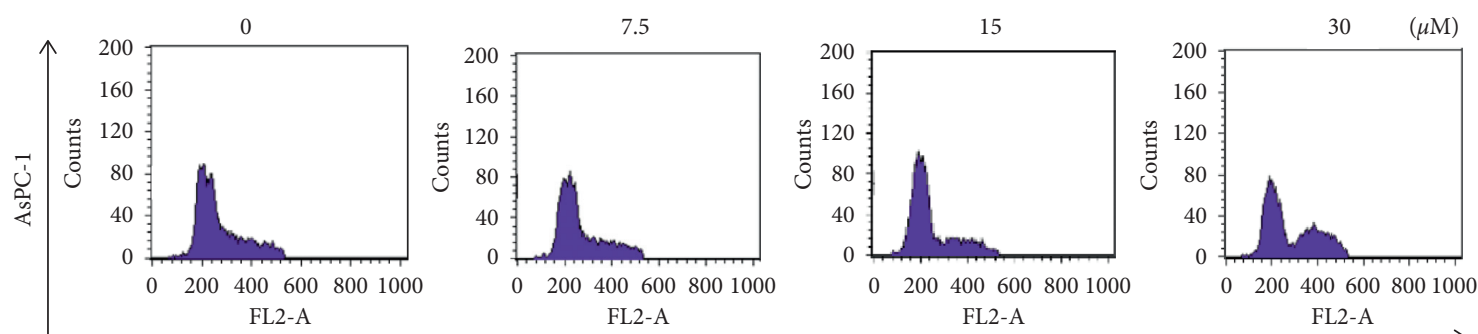

\begin{tabular}{|c|c|c|c|c|}
\hline $\operatorname{MSB}(\mu \mathrm{M})$ & 0 & 7.5 & 15 & 30 \\
\hline Sub-G1 & $0.40 \pm 0.04$ & $0.61 \pm 0.36$ & $0.86 \pm 0.28$ & $1.23 \pm 0.12^{* *}$ \\
\hline G1 & $62.79 \pm 3.80$ & $64.80 \pm 3.09$ & $75.60 \pm 0.62$ & $60.74 \pm 0.88$ \\
\hline G1-S & $22.41 \pm 4.55$ & $19.80 \pm 4.31$ & $10.88 \pm 0.95$ & $15.01 \pm 1.51$ \\
\hline G2-M & $11.68 \pm 1.12$ & $12.98 \pm 1.52$ & $10.98 \pm 1.14$ & $18.81 \pm 0.56^{* *}$ \\
\hline
\end{tabular}

(d)

Figure 1: Misaponin B exerts cytotoxicity in several cancer cells, such as A549, H460, AsPC-1, and SKOV3 cancer cells and induces G2/M arrest in A549 cells and AsPC-1 cells. (a) Chemical structure of Misaponin B (MSB). (b) Cytotoxicity of Misaponin B in several cancer cells. MTT assay was used to evaluate the cytotoxicity of Misaponin B in A549, H460, AsPC-1, and SKOV3 cells. (c, d) Effect of Misaponin B on G2/M arrest in A549 and AsPC-1 cells by FACS analysis. Data are represented as means \pm SD of two independent experiments. ${ }^{*} p<0.05$; ${ }^{* *} p<0.01 ;{ }^{* * *} p<0.001$ vs untreated control.

2.9. Transmission Electron Micrograph (TEM). A549 cells were treated with Misaponin B for $24 \mathrm{~h}$ and fixed for $12 \mathrm{~h}$ in $2 \%$ glutaraldehyde - paraformaldehyde in $0.1 \mathrm{M}$ phosphate buffer $(\mathrm{pH}$ 7.4) and washed in $0.1 \mathrm{M}$ phosphate buffer and were postfixed with $1 \% \mathrm{OsO}_{4}$ in $0.1 \mathrm{M}$ phosphate buffer for $2 \mathrm{~h}$ and dehydrated in an ascending gradual series (50 100\%) of ethanol and infiltrated with propylene oxide. After pure, fresh resin embedding and polymerization at $65^{\circ} \mathrm{C}$ on a electron microscope oven (TD-700, DOSAKA, Japan) for $24 \mathrm{~h}$, thick sections were 


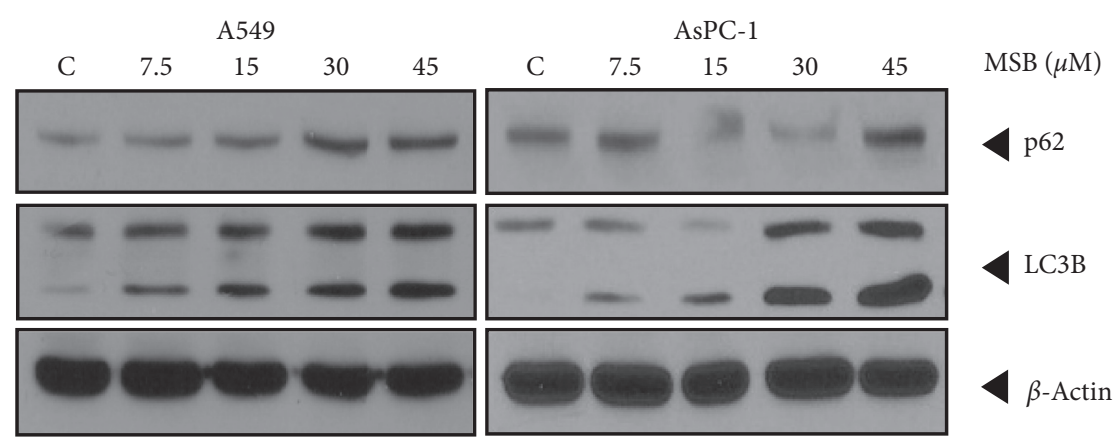

(a)
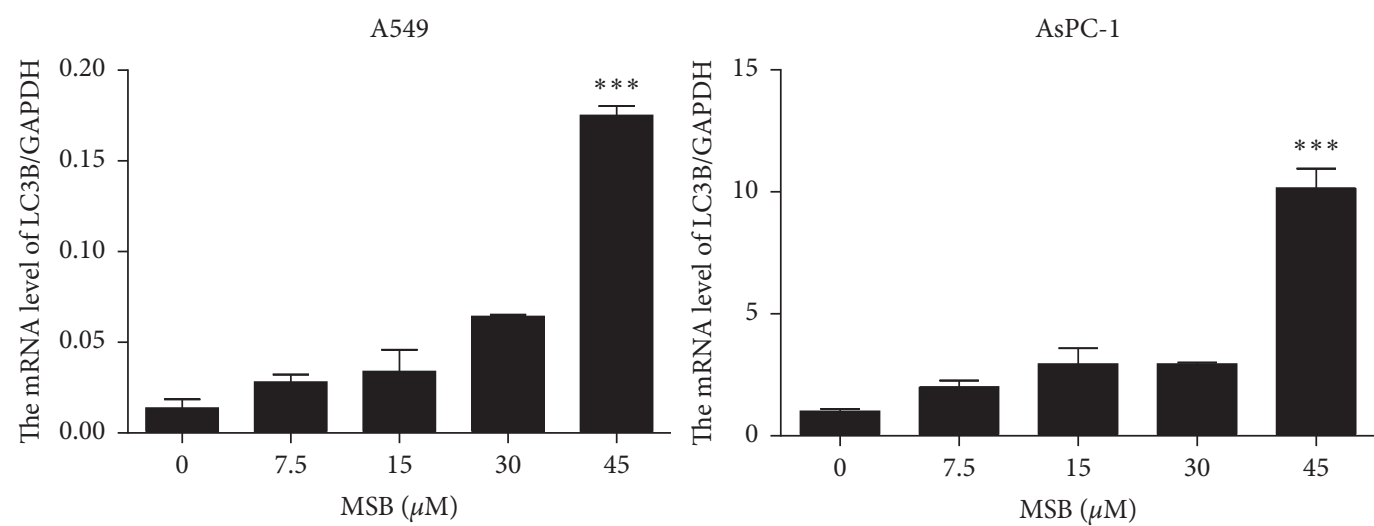

(b)

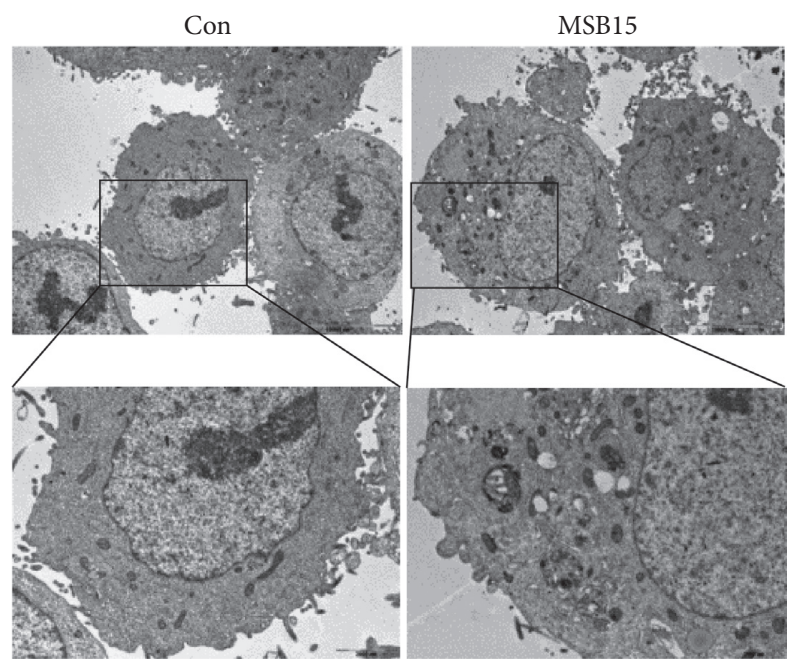

(c)

FIGURE 2: (a) Effect of Misaponin B on LC3B conversion and p62 at the protein level in A549 and AsPC-1 cells. Western blot analysis of A549 and AsPC-1 cells were treated with Misaponin B (7.5 to $45 \mu \mathrm{M})$ for $24 \mathrm{~h}$ and subjected to western blotting $(n=2)$. (b, c) Effect of Misaponin B on LC3B at mRNA level. A549 and AsPC-1 cells were treated with Misaponin B for $24 \mathrm{~h}$ and subjected to RT-PCR analysis. (d) Transmission electron microscopy (TEM) images showing autophagic vacuoles in Misaponin B-treated A549 cells. Misaponin B-treated A549 cells were photographed by TEM $(n=3)$.

cut and stained with toluidine blue (Sigma, T3260, USA) and were double marked with 6\% uranyl acetate (EMS, 22400 for $20 \mathrm{~min}$ ) and lead citrate (for $10 \mathrm{~min}$ ) for contrast staining. All the pieces were observed by transmission electron microscopy (JEM-1011, Japan) at the voltage of $80 \mathrm{kV}$. The grid is removed, blotted with a filter paper, and positioned onto a drop of $2 \%$ uranyl acetate for $15 \mathrm{sec}$. After removing the excess uranyl acetate, the EM grid was photographed for transmission electron microscopy (JEM1011, Japan). A549 cells were randomly selected and imaged at 4,000x or 10,000x magnification by transmission electron microscopy.

2.10. Statistical Analysis. GraphPad Prism 5.0 software was used for statistical analyses. Data were analyzed using one- 


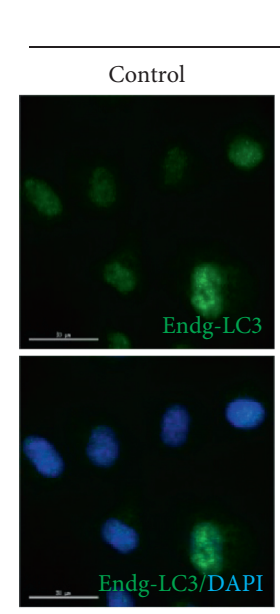

Control
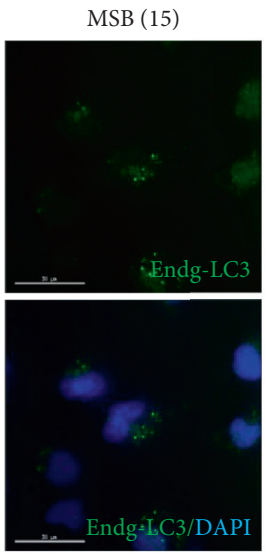

(a)

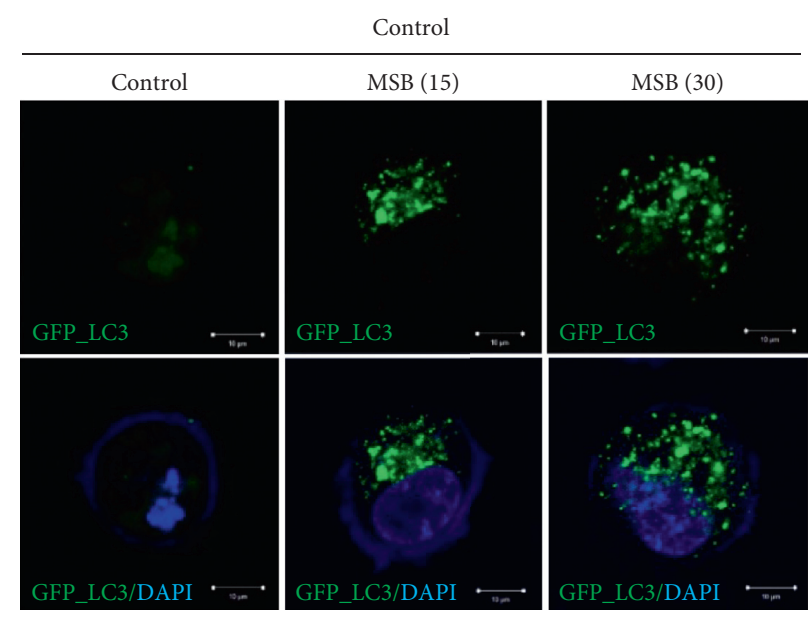

(b)

Figure 3: Misaponin B increased the number of LC3-positive fluorescent punctae and autophagosomes in A549 cells. (a) Misaponin B increased the punctae of endogenous LC3B in A549 cells. Immunostaining was performed with LC3 antibody in Misaponin B-treated A549 cells. (b) Misaponin B increased the autophagosome formation in GFP-LC3 expressing A549 cells. After transfection with GFP-LC3 construct, A549 cells were exposed to Misaponin B for $24 \mathrm{~h}$ and then visualized by confocal microscopy $(n=2)$.

way ANOVA or two-way ANOVA. $p<0.05$ was considered statistically significant.

\section{Results}

3.1. Misaponin B Induced Cytotoxicity and G2/Arrest in A549 Cells. To investigate the cytotoxic effect of Misaponin B (Figure 1(a)), different concentrations of Misaponin B (7.5, $15,30,45,60,120 \mu \mathrm{M}$ ) were added for $24 \mathrm{~h}$ in NSCLC (A549 and H460), pancreatic cancer AsPC-1, and ovarian cancer SKOV3 cells. Misaponin B reduced viability in a concentration-dependent manner in A549, H460, AsPC-1, and SKOV3 (Figure 1(b)). A549 and AsPC-1 cells were most susceptible to Misaponin B among the treated cancer cells. Next, cell cycle analysis was performed following PI staining by FACS. Here, Misaponin B induced G2/M arrest in A549 and AsPC-1 cells (Figures 1(c) and 1(d)).

\subsection{Misaponin B Induced Cytokinesis Failure in A549 Cells.} Cytokinesis was evaluated in Misaponin B-treated A549 cells. Briefly, A549 cells were treated with different concentrations $(15,30 \mu \mathrm{M})$ of Misaponin and stained with DAPI for immunofluorescence. As shown in Figures 2(a) and 2(b), a fraction of $\alpha$-tubulin was localized to the centrosomes, but not moved to the cytokinesis for mitotic cell division.

\subsection{Misaponin B Increased Accumulation of Autophagic} Marker LC3B in a Concentration-Dependent Manner in A549 and AsPC-1 Cells. To evaluate the effect of Misaponin B on autophagy, western blotting was conducted in A549 and AsPC-1 cells. Misaponin B increased LC3B conversion and p62/SQSTM1 accumulation at the protein level in a concentration-dependent fashion in the A549 or AsPC-1 cells (Figure 2(a)). Consistently, qRT-PCR analysis revealed that the mRNA expression level of LC3B was increased in Misaponin B-treated A549 or AspC-1 cells (Figures 2(b) and 2(c)). TEM is one of the standard methods to detect autophagy [25]. TEM observation revealed that the number of autophagosomes was observed in the cytoplasm of A549 cells treated by Misaponin B (Figure 2(d)).

3.4. Misaponin B Increased the Number of LC3-Positive Fluorescent Punctae and the Formation of Autophagosomes in Misaponin B-Treated A549 Cells. Misaponin B increased the punctae stained with endogenous LC3 (endog LC3) and DAPI in A549 cells compared to untreated controls (Figure 3(a)). Consistently, the number of green GFP-LC3 autophagosomes was significantly increased in Misaponin B-treated A549 cells compared to untreated control (Figure 3(b)).

3.5. Mi-saponin B Inhibited Autophagy Flux in A549 Cells. To investigate whether or not Misaponin B induces autophagic flux, A549 cells were transfected with a tandem RFPGFP-LC3 construct and then exposed to Misaponin B (15 and $30 \mu \mathrm{M}$ ) for $24 \mathrm{~h}$. Then, autophagy flux was evaluated in A549 cells by using confocal microscopy analysis. Misaponin $B$ exhibited yellow color punctae by merging image in RFPGFP-LC3 construct-transfected A549 cells (Figure 4).

\section{Discussion}

The current study was undertaken to elucidate the underlying antitumor mechanism of Misaponin B in A549 and AsPC-1 cancer cells. Herein, Misaponin B exerted cytotoxicity in A549, H460 nonsmall cell lung cancer, SKOV3 ovarian cancer, and AsPC-1 pancreatic cancer cells, implying significant cytotoxic effect of Misaponin B in several cancers.

To check the type of cell death by Misaponin B in A549 and AsPC-1 cells, cell cycle analysis and western blotting were performed. Here, Misaponin B did not induce PARP 
MSB $(\mu \mathrm{M})$

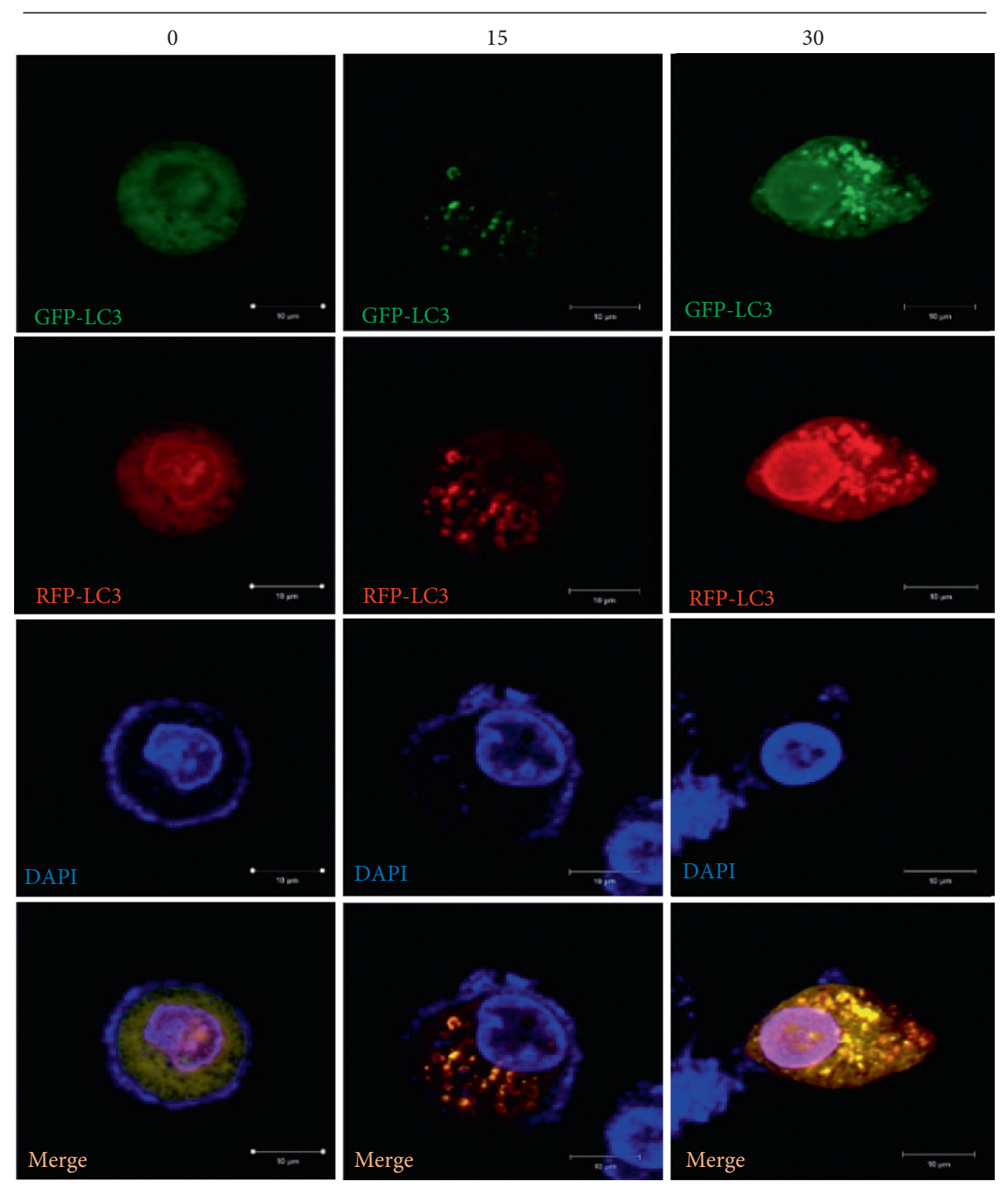

Figure 4: Misaponin B disturbed autophagic flux in A549 cells. After transfection with RFP-GFP-LC3 plasmid, A549 cells were treated by Misaponin B and visualized by confocal microscopy. Representative fluorescence images showing the red/green signals from the mRFPGFP-LC3 construct for measuring the autophagic flux. Nuclei were stained with DAPI (blue). Punctae: RFP+GFP+ (R+G+: early autophagosomes), RFP+GFP- (R+G-: autolysosomes), and total LC3 punctae. Images merged red and green channels; scale bar: $10 \mu \mathrm{M}$ $(n=2)$.

cleavages up to $40 \mu \mathrm{M}$ (Supplementary Figure 1), but cell cycle analysis revealed that Misaponin B induced G2/M arrest and no sub-G1 accumulation in A549 and AsPC-1 cells, indicating the cytotoxicity of Misaponin B can be induced by $\mathrm{G} 2 / \mathrm{M}$ arrest, not apoptosis signaling, in A549 and AsPC-1 cells.

Autophagy is the catabolic process for the recycling of macromolecules and the degradation of long-lived proteins, pathogens, damaged DNA elements, and damaged organelles [26, 27]. Growing evidences have demonstrated that impaired autophagy by blocking of fusion of autophagosome and lysosome would rather cause cell death due to over consumption of energy to survive [28]. It is well known that autophagic cell death can be induced, only when apoptosis is blocked by caspase inhibitor or lacking Bax and Bak expression in cells $[29,30]$. In the current study, Misaponin $B$ increased the conversion of LC3B and green GFP-LC3 punctae as a typical autophagy marker by western blotting, qRT-PCR, and immunofluorescence in A549 cells, demonstrating the potent autophagy induction by Misaponin B in A549 and AsPC-1 cells. Autophagy flux assay is frequently employed to assess autophagy degradation process in the cells [31]. Usually, GFP and mRFP signals are detected by merging image fluorescence before fusion with lysosomes, while only the mRFP signal is detected by merging image red fluorescence after fusion with lysosomes for complete autophagy flux [32-34]. The green-red/yellow punctae indicate autophagosomes, whereas the autolysosome (autophagosome fused to lysosome) would be red in color [35]. Here, autophagy flux assay using GFP-mRFP-LC3 construct 
transfection showed that Misaponin B increased merged yellow GFP-LC3 punctae in A549 cells, implying that Misaponin B impaired autophagy to induce autophagic cell death in A549 cells. Likewise, previous evidences revealed that arsenic trioxide treatment of malignant glioma cells induced G2/M arrest and autophagic cell death [19], and plumbagin inhibited proliferation to undergo G2/M arrest and autophagic cell death [36]. However, for detailed mechanistic study, further molecular studies including PPI, luciferase assay, and animal study are required with Misaponin $\mathrm{B}$ in the near future.

\section{Conclusions}

In summary, Misaponin B exerted significant cytotoxicity, induced G2/M arrest, and increased the expression of LC3B, a maker of autophagy, at the protein and mRNA levels in A549 cells. Furthermore, Misaponin B increased autophagic vacuoles GFP-LC3 punctae in A549 cells, but Misaponin B disturbed autophagy flux by revealing merged yellow GFPLC3 punctae. Overall, our findings support evidences that Mi-saponin B induces G2/M arrest and autophagic cell death as a potent anticancer agent.

\section{Data Availability}

The data and materials supporting the conclusions of this article are included within the article.

\section{Conflicts of Interest}

The authors have no conflicts of interest to declare.

\section{Authors' Contributions}

Gunho Won, Ji Hoon Jung, and Eun Jung Sohn contributed equally.

\section{Acknowledgments}

The authors thank the Kim lab members for active discussion and suggestions. Sung-Hoon Kim was supported in part by the National Research Foundation of Korea (NRF) Grant funded by the Korea Government (MEST) (no. 2017R1A2A1A17069297).

\section{References}

[1] K. Arnold, "Statistics offer insights into progress against cancer," CancerSpectrum Knowledge Environment, vol. 95, no. 17, pp. 1266-1267, 2003.

[2] A. Kamb, S. Wee, and C. Lengauer, "Why is cancer drug discovery so difficult?," Nature Reviews Drug Discovery, vol. 6, no. 2, pp. 115-120, 2007.

[3] C. C. Earle, J. S. Tsai, R. D. Gelber, M. C. Weinstein, P. J. Neumann, and J. C. Weeks, "Effectiveness of chemotherapy for advanced lung cancer in the elderly: instrumental variable and propensity analysis," Journal of Clinical Oncology, vol. 19, no. 4, pp. 1064-1070, 2001.

[4] L. S. Rosen, D. Gordon, M. Kaminski et al., "Long-term efficacy and safety of zoledronic acid compared with pamidronate disodium in the treatment of skeletal complications in patients with advanced multiple myeloma or breast carcinoma," Cancer, vol. 98, no. 8, pp. 1735-1744, 2003.

[5] Y. Li, W. Sun, N. Han, Y. Zou, and D. Yin, "Curcumin inhibits proliferation, migration, invasion and promotes apoptosis of retinoblastoma cell lines through modulation of miR-99a and JAK/STAT pathway," BMC Cancer, vol. 18, no. 1, p. 1230, 2018.

[6] J. Kim, M. Yun, E.-O. Kim et al., "Decursin enhances TRAILinduced apoptosis through oxidative stress mediated- endoplasmic reticulum stress signalling in non-small cell lung cancers," British Journal of Pharmacology, vol. 173, no. 6, pp. 1033-1044, 2016.

[7] J. Jang, S. J. Jeong, H. Y. Kwon et al., "Decursin and doxorubicin are in synergy for the induction of apoptosis via STAT3 and/or mTOR pathways in human multiple myeloma cells," Evid Based Complement Alternat Med, vol. 2013, Article ID 506324, 13 pages, 2013.

[8] S. W. Ryter, D. Bhatia, and M. E. Choi, "Autophagy: a lysosome-dependent process with implications in cellular redox homeostasis and human disease," Antioxidants \& Redox Signaling, vol. 30, no. 1, pp. 138-159, 2019.

[9] L. Gao, C. E. Jauregui, and Y. Teng, "Targeting autophagy as a strategy for drug discovery and therapeutic modulation," Future Medicinal Chemistry, vol. 9, no. 3, pp. 335-345, 2017.

[10] N. Mizushima, B. Levine, A. M. Cuervo, and D. J. Klionsky, "Autophagy fights disease through cellular self-digestion," Nature, vol. 451, no. 7182, pp. 1069-1075, 2008.

[11] B. Levine, "Autophagy and cancer," Nature, vol. 446, no. 7137, pp. 745-747, 2007.

[12] M. M. Hippert, P. S. O’Toole, and A. Thorburn, “Autophagy in cancer: good, bad, or both?: figure 1," Cancer Research, vol. 66, no. 19, pp. 9349-9351, 2006.

[13] Z. Xie and D. J. Klionsky, "Autophagosome formation: core machinery and adaptations," Nature Cell Biology, vol. 9, no. 10, pp. 1102-1109, 2007.

[14] K. Nihira, Y. Miki, K. Ono, T. Suzuki, and H. Sasano, "An inhibition of p62/SQSTM1 caused autophagic cell death of several human carcinoma cells," Cancer Science, vol. 105, no. 5, pp. 568-575, 2014.

[15] X. Li, W. Xie, C. Xie et al., "Curcumin modulates miR-19/ $\mathrm{PTEN} / \mathrm{AKT} / \mathrm{p} 53$ axis to suppress bisphenol A-induced MCF7 breast cancer cell proliferation," Phytotherapy Research, vol. 28, no. 10, pp. 1553-1560, 2014.

[16] T. Shintani and D. J. Klionsky, "Autophagy in health and disease: a double-edged sword," Science, vol. 306, no. 5698, pp. 990-995, 2004.

[17] Y. Kondo, T. Kanzawa, R. Sawaya, and S. Kondo, "The role of autophagy in cancer development and response to therapy," Nature Reviews Cancer, vol. 5, no. 9, pp. 726-734, 2005.

[18] S. Paglin, T. Hollister, T. Delohery et al., "A novel response of cancer cells to radiation involves autophagy and formation of acidic vesicles," Cancer Research, vol. 61, no. 2, pp. 439-444, 2001.

[19] T. Kanzawa, Y. Kondo, H. Ito, S. Kondo, and I. Germano, "Induction of autophagic cell death in malignant glioma cells by arsenic trioxide," Cancer Research, vol. 63, no. 9, pp. 2103-2108, 2003.

[20] C. Bachran, S. Bachran, M. Sutherland, D. Bachran, and H. Fuchs, "Saponins in tumor therapy," Mini-Reviews in Medicinal Chemistry, vol. 8, no. 6, pp. 575-584, 2008.

[21] S. Man, W. Gao, Y. Zhang, L. Huang, and C. Liu, "Chemical study and medical application of saponins as anti-cancer agents," Fitoterapia, vol. 81, no. 7, pp. 703-714, 2010. 
[22] Y.-Z. Zhao, Y.-Y. Zhang, H. Han et al., "Advances in the antitumor activities and mechanisms of action of steroidal saponins," Chinese Journal of Natural Medicines, vol. 16, no. 10, pp. 732-748, 2018.

[23] K. Yoshikawa, M. Tanaka, S. Arihara et al., "New oleanene triterpenoid saponins fromMadhucalongifolia," Journal of Natural Products, vol. 63, no. 12, pp. 1679-1681, 2000.

[24] S. E. Won GH, M. S. Jeong, S. W. Yoon, J. Lee, B. L. Kim, and S. H. Kim, "Misaponin B induces G2-M arrest and autophagy via upregulation of miR1290 in non-small cell lung cancer (NSCLC) A549 cells," in Proceedings of the 106th Annual Meeting of the American Association for Cancer Research, Philadelphia, PA, USA, April 2015.

[25] D. J. Klionsky, H. Abeliovich, P. Agostinis et al., "Guidelines for the use and interpretation of assays for monitoring autophagy in higher eukaryotes," Autophagy, vol. 4, no. 2, pp. 151-175, 2008.

[26] D. A. Gewirtz, "Autophagy and senescence in cancer therapy," Journal of Cellular Physiology, vol. 229, no. 1, pp. 6-9, 2014.

[27] K. Sharma, R. Goehe, J. M. Beckta, K. Valerie, and D. A. Gewirtz, "Autophagy and radiosensitization in cancer," EXCLI Journal, vol. 13, no. 13, pp. 178-191, 2014.

[28] G. Mariño, M. Niso-Santano, E. H. Baehrecke, and G. Kroemer, "Self-consumption: the interplay of autophagy and apoptosis," Nature Reviews Molecular Cell Biology, vol. 15, no. 2, pp. 81-94, 2014.

[29] L. Xue, G. C. Fletcher, and A. M. Tolkovsky, "Mitochondria are selectively eliminated from eukaryotic cells after blockade of caspases during apoptosis," Current Biology, vol. 11, no. 5, pp. 361-365, 2001.

[30] Y. Xu, S. O. Kim, Y. Li, and J. Han, "Autophagy contributes to caspase-independent macrophage cell death," Journal of Biological Chemistry, vol. 281, no. 28, pp. 19179-19187, 2006.

[31] U. M. Nazim and S. Y. Park, "Attenuation of autophagy flux by 6 -shogaol sensitizes human liver cancer cells to TRAILinduced apoptosis via p53 and ROS," International Journal of Molecular Medicine, vol. 43, no. 2, 2018.

[32] X.-J. Zhang, S. Chen, K.-X. Huang, and W.-D. Le, "Why should autophagic flux be assessed?," Acta Pharmacologica Sinica, vol. 34, no. 5, pp. 595-599, 2013.

[33] J. Klose, M. V. Stankov, M. Kleine et al., "Inhibition of autophagic flux by salinomycin results in anti-cancer effect in hepatocellular carcinoma cells," PLoS One, vol. 9, no. 5, Article ID e95970, 2014.

[34] N. Furuta, N. Fujita, T. Noda, T. Yoshimori, and A. Amano, "Combinational soluble N-ethylmaleimide-sensitive factor attachment protein receptor proteins VAMP8 and Vtilb mediate fusion of antimicrobial and canonical autophagosomes with lysosomes," Molecular Biology of the Cell, vol. 21, no. 6, pp. 1001-1010, 2010.

[35] K. C. Yang, P. Sathiyaseelan, C. Ho, and S. M. Gorski, "Evolution of tools and methods for monitoring autophagic flux in mammalian cells," Biochemical Society Transactions, vol. 46, no. 1, pp. 97-110, 2018.

[36] P.-L. Kuo, Y.-L. Hsu, and C.-Y. Cho, "Plumbagin induces G2$\mathrm{M}$ arrest and autophagy by inhibiting the AKT/mammalian target of rapamycin pathway in breast cancer cells," Molecular Cancer Therapeutics, vol. 5, no. 12, pp. 3209-3221, 2006. 